\title{
Inhibición de la corrosión del acero ASTM A192 en solución de ácido clorhídrico por el mucilago de Linum usitatissimum
}

\author{
Inhibition of corrosion of ASTM A192 steel in \\ hydrochloric acid solution by the mucilage of \\ Linum usitatissimum
}

\author{
Nilthon Emerson Zavaleta-Gutierrez ${ }^{1}$, Elmer Rolando Polo-Briceño ${ }^{1}$, \\ Raúl Siche ${ }^{2}$, Luis Manuel Angelats-Silva ${ }^{3}$
}

\footnotetext{
${ }^{1}$ Departamento de Minas y Metalurgia, Facultad de Ingeniería, Universidad Nacional de Trujillo, Av. Juan Pablo II s/n, Ciudad Universitaria, Trujillo, Trujillo,Perú. e-mail:nzavaleta@unitru.edu.pe,e.polo.14@gmail.com

${ }^{2}$ Facultad de Ciencias Agropecuarias, Universidad Nacional de Trujillo, Av. Juan Pablo II s/n, Ciudad Universitaria, Trujillo, Trujillo, Perú. e-mail: rsiche@unitru.edu.pe

${ }^{3}$ Laboratorio de Investigación Multidisciplinaria, Universidad Privada Antenor Orrego, Av. América Sur 3145, Trujillo, Trujillo, Perú.

e-mail: langelatss@upao.edu.pe
}

\section{RESUMEN}

En este estudio se investigó la inhibición de la corrosión del acero ASTM A192 en HCl 0,5 M a diferentes temperaturas, por el mucílago de Linum usitatissimum, mediante las técnicas de extrapolación Tafel, resistencia a la 0 , espectroscopia de impedancia electroquímica y modulación de frecuencia electroquímica.

El mucílago de Linum actúa como un óptimo inhibidor de la corrosión del acero ASTM A192 en HCl. La eficiencia de inhibición promedio obtenidos con las técnicas evaluadas fueron de $84,3 \%$ a $25{ }^{\circ} \mathrm{C}, 89,0 \%$ a $45^{\circ} \mathrm{C}$ y $91,8 \%$ a $65{ }^{\circ} \mathrm{C}$, con una concentración del mucílago de $1 \mathrm{~g} / \mathrm{l}$. Se encontró que la eficiencia de inhibición y la constante de adsorción incrementaron con el aumento de la temperatura. La entalpía estándar de adsorción fue positiva y la energía de activación aparente disminuyó con el aumento de la concentración del mucílago. Todos estos parámetros indican que la adsorción de mucílago en el acero evaluado se produce a través de una adsorción química. Además, conforme incrementa la temperatura, el comportamiento del mucílago cambia desde un inhibidor tipo anódico a un inhibidor de tipo mixto.

Palabras clave: Linum usitatissimum, acero ASTM A192, inhibición ácida, EIS, EFM.

\begin{abstract}
In this study, the inhibition of the corrosion of ASTM A192 steel in HCl 0,5 M at temperature different, by the Linum usitatissimum mucilage, was investigated using techniques: Tafel extrapolation, linear polarization resistance, electrochemical impedance spectroscopy and electrochemical frequency modulation.

The Linum mucilage acts as an optimum corrosion inhibitor of ASTM A192 steel in HCl. The average inhibition efficiency obtained with the evaluated techniques were of $84,3 \%$ at $25{ }^{\circ} \mathrm{C}, 89,0 \%$ at $45{ }^{\circ} \mathrm{C}$ and $91,8 \%$ at $65{ }^{\circ} \mathrm{C}$, with a concentration of the mucilage of $1 \mathrm{~g} / \mathrm{l}$. It was found that the efficiency of inhibition and the adsorption constant increased with increasing temperature. The standard enthalpy of adsorption was positive and the apparent activation energy decreased with the increase of the concentration of the mucilage. All these parameters indicate that the adsorption of flaxseed mucilage in steel evaluated occurs through a chemical adsorption. In addition, as the temperature increases, mucilage behavior changes from an anodic inhibitor to a mixed-type inhibitor.
\end{abstract}

Keywords: Linum usitatissimum, ASTM A192 steel, acid inhibition, EIS, EFM. 


\section{INTRODUCCIÓN}

Es ampliamente reconocida la alta eficiencia a la inhibición de la corrosión que presentan los inhibidores orgánicos sintéticos en las industrias de limpieza química, decapado ácido, acidificación de pozos de petróleo y desincrustación ácida; sin embargo, esto genera efluentes con una de alta toxicidad para el medio ambiente. En la actualidad, las regulaciones ambientales obligan a tomar conciencia sobre su uso y buscar alternativas más ecológicas. Por ello, en los últimos años las investigaciones en corrosión están orientados al desarrollo de inhibidores de corrosión (IC) a base de extractos de planta (EP), que son compuestos con alta eficiencia de inhibición (EI) y con bajo riesgo de contaminación del medio ambiente.

El uso exitoso de sustancias de origen natural para inhibir la corrosión de aceros al carbono en un medio de $\mathrm{HCl}$ ha sido reportado por varios investigadores, como lo muestran los artículos de revisión de IC verdes. RAJA y SETHURAMAN [1] realizaron una revisión de alrededor de 60 estudios de EP evaluados como IC, concluyendo que pocas veces se realiza el análisis fitoquímico del EP, desconociéndose el componente activo responsable de la inhibición. SANGEETHA et al. [2] analizaron 111 investigaciones de EP como IC, mostrando las condiciones de ensayo, las técnicas utilizadas y sus características de inhibición. PATNI et al.

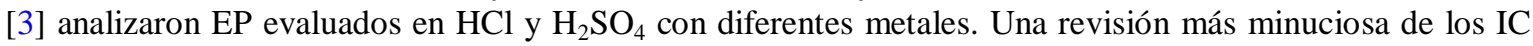
para aceros en medio ácido lo realizaron FINSGAR y JACKSON [4].

No obstante, la gran cantidad de estudios de EP como inhibidores de la corrosión del acero en $\mathrm{HCl}$, no se ha reportado estudios con el Linum usitatissimum. La semilla del Linum usitatissimun contiene una alta cantidad de fibra soluble (9-10\%) [5], conocida como mucílago de Linum (ML), cuyo rendimiento de extracción es mayor en agua caliente [6]. El ML incluye dos componentes de polisacáridos, neutros y ácidos. La fracción neutra contiene L-arabinosa, D-xilosa y D-galactosa y la fracción ácida contiene L-ramnosa, Lfucosa, L-galactosa y ácido D-galacturónico [7]. Estos polisacáridos contienen heteroátomos de O, que refuerzan su propiedad de adsorción sobre la superficie de metal.

Así, el objetivo de este estudio fue evaluar el efecto inhibidor del mucílago de Linum usitatissimum en $\mathrm{HCl}$ 0,5 M a diferentes temperaturas en la corrosión del acero ASTM A192, utilizando las técnicas de extrapolación Tafel, resistencia a la polarización lineal, espectroscopia de impedancia electroquímica y modulación de frecuencia electroquímica.

\section{MATERIALES Y MÉTODOS}

\subsection{Materiales}

Para la obtención del ML se utilizó semillas de Linum usitatissimum procedente del distrito Chugay de la Provincia Sánchez Carrión, Perú. Los ensayos de corrosión se realizaron con electrodos de trabajo fabricados desde un tubo sin costura del acero ASTM A192; este acero ferrítico-perlítico es utilizado en los tubos de las calderas de las centrales termoeléctricas convencionales. En la tabla 1 se muestra su composición química.

Tabla 1: Composición química del acero ASTM A192 (\% en peso).

\subsection{Extracción del mucilago de linum}

\begin{tabular}{c|c|c|c|c|c}
\hline $\mathbf{C}$ & $\mathbf{S i}$ & $\mathbf{M n}$ & $\mathbf{P}$ & $\mathbf{S}$ & $\mathbf{C u}$ \\
\hline 0,14 & 0,16 & 0,53 & 0,008 & 0,024 & 0,21 \\
\hline
\end{tabular}

El ML se extrajo mediante un procedimiento acuoso usando agua bidestilada a presión atmosférica y un $\mathrm{pH}$ de 7,0 [6]. La temperatura de extracción fue $80^{\circ} \mathrm{C}$, con una relación de agua $(\mathrm{ml}) / \mathrm{semilla}(\mathrm{g})$ de $20: 1$, por un tiempo de 1 h y sin agitación. Seguidamente se realizó la separación de la solución conteniendo el mucílago mediante una malla de $250 \mu \mathrm{m}$, y pasó directamente a su evaporación en una estufa a $100{ }^{\circ} \mathrm{C}$. El ML deshidratado fue pulverizado y ensayado con un espectrofotómetro de Infrarrojo con transformada de Fourier, marca Thermo Scientific, modelo Nicolet iS50, equipado con una celda de reflectancia total atenuada de $\mathrm{ZnSe}$.

\subsection{Ensayos electroquímicos}

Los ensayos electroquímicos fueron realizados en una celda convencional de tres electrodos de vidrio de 150 $\mathrm{cm}^{3}$ de capacidad. La celda consistió de un electrodo de trabajo cilíndrico del acero A192 con un área expuesta de alrededor de $2,0 \mathrm{~cm}^{2}$, un electrodo de calomel saturado como electrodo de referencia, una varilla de grafito como contraelectrodo y un termómetro para la medición de la temperatura. Un capilar de Luggin fue incluido en el diseño, acondicionado cerca de la superficie del electrodo de trabajo para minimizar la caída IR. Las soluciones de $\mathrm{HCl}$ 0,5 M se prepararon de manera fresca por disolución del $\mathrm{HCl} 36 \%$ con agua bidestila- 
da, al cual se le adicionó el ML en proporciones de 0,01, 0,05, 0,10, 0,50 y 1,00 g/l. Previo a los ensayos de corrosión, el electrodo de trabajo (acero A192) fue desbastado sucesivamente con papeles de carburo de silicio de diferentes grados (220 \# - 1000 \#), desengrasado con acetona, lavado con agua bidestilada y finalmente secado.

Todos los ensayos electroquímicos se realizaron por triplicado utilizando el potenciostato Gamry Reference 3000. El electrodo de trabajo se mantuvo por un tiempo de 60 minutos en las soluciones ácidas antes del inicio de las mediciones para lograr un valor cuasi estacionario para el potencial de circuito abierto.

Las curvas de polarización potenciodinámicas se realizaron con una velocidad de barrido de $0,1 \mathrm{mV} / \mathrm{s}$. El rango de potencial evaluado fue de $\pm 0,25 \mathrm{~V}$ respecto al potencial de circuito abierto ( $\left.\mathrm{E}_{\text {corr }}\right)$

Las mediciones de modulación por frecuencia electroquímica se realizaron con la aplicación de una señal de perturbación de $10 \mathrm{mV}$ de amplitud respecto al potencial de circuito abierto y con dos frecuencias de 2 y $5 \mathrm{~Hz}$. Con los datos de densidades de corriente y frecuencias se graficaron los espectros de intermodulación y mediante el software EFM 140 se obtuvieron la densidad de corriente de corrosión ( $\mathrm{i}_{\text {corr }}$ ), las pendientes de Tafel $\left(\beta_{\mathrm{c}}\right.$ y $\beta_{\mathrm{a}}$ ) y los factores de causalidad (CF1 y CF2) [8]. Considerando que la densidad de corriente de corrosión es directamente proporcional a la velocidad de corrosión $\left(\mathrm{V}_{\text {corr }}\right)$, la eficiencia de inhibición, EI (\%), fue calculada desde los valores medidos de $\mathrm{i}_{\text {corr }}$ utilizando la ecuación:

$$
E I(\%)=100\left[\frac{\left(i_{\text {corr }}^{o}-i_{\text {corr }}^{i n h}\right)}{i_{\text {corr }}^{o}}\right]
$$

donde $i_{\text {corr }}^{\mathrm{o}} \mathrm{y} \mathrm{i}_{\text {corr }}^{\mathrm{inh}}$ son la densidad de corriente de corrosión para las soluciones sin inhibidor y con inhibidor, respectivamente. Todos los valores de $\mathrm{i}_{\text {corr }}$ fueron convertidos a velocidad de corrosión $\left(\mathrm{V}_{\text {corr }}\right)$ en mm.año ${ }^{-1}$ según la ecuación:

$$
V_{\text {corr }}\left[\frac{m m}{a \tilde{a} o}\right]=3267,98\left[\frac{i_{c o r r} \cdot P_{e q}}{\rho}\right]
$$

donde $\mathrm{i}_{\text {corr }}$, es la densidad de corriente de corrosión $\left(\mathrm{A} / \mathrm{cm}^{2}\right) ; \mathrm{P}_{\mathrm{eq}}$ es la masa equivalente del hierro $(27,925 \mathrm{~g})$, y $\rho$ es la densidad del acero A192 $\left(7,86 \mathrm{~g} / \mathrm{cm}^{3}\right)$.

Para las mediciones de resistencia a la polarización lineal, se utilizó un barrido desde $\pm 20 \mathrm{mV}$ respecto al potencial de circuito abierto a una velocidad de $0,1 \mathrm{mV} / \mathrm{s}$, y la resistencia a la polarización $\left(\mathrm{R}_{\mathrm{p}}\right)$ se obtuvo a partir de la pendiente de la curva $\mathrm{E}$ vs $\mathrm{i}$ en las proximidades del $\mathrm{E}_{\text {corr }}$, mediante el software DC105 de Gamry. Considerando que $1 / R_{p}$ es directamente proporcional a la velocidad de corrosión, las eficiencias de inhibición del ML fueron calculadas a partir de valores de $\mathrm{R}_{\mathrm{p}}$ obtenidos desde los datos de polarización lineal con las diferentes concentraciones del ML, usando la ecuación:

$$
E I(\%)=100\left[\frac{R_{p(\text { inh })}-R_{p}}{R_{p(\text { inh })}}\right]
$$

donde $\mathrm{R}_{\mathrm{p}}$ y $\mathrm{R}_{\mathrm{p} \text { (inh) }}$ son los valores de resistencia a la polarización sin y con inhibidor, respectivamente. Para el cálculo de la densidad de corriente de corrosión $\left(\mathrm{A} / \mathrm{cm}^{2}\right)$ se utilizó la ecuación de Stern-Geary:

$$
i_{\text {corr }}=\frac{\beta_{a} \cdot \beta_{c}}{2,303\left(\beta_{a}+\beta_{c}\right) R_{p}}
$$

donde, $\beta_{\mathrm{a}}$ y $\beta_{\mathrm{c}}$ son las pendientes de Tafel anódica y catódica (V/década) obtenidas desde los ensayos de modulación de frecuencia electroquímica, y $R_{p}$ es la resistencia a la polarización $\left(\Omega . \mathrm{cm}^{2}\right)$. La $\mathrm{V}_{\text {corr }}$ en (mm/año) se obtuvo mediante la ecuación (2).

Las mediciones de espectroscopia de impedancia electroquímica se realizaron utilizando señales de corriente alterna de amplitud de $10 \mathrm{mV}$ respecto al potencial de circuito abierto en el rango de frecuencias de $10000 \mathrm{~Hz}$ hasta $0,1 \mathrm{~Hz}$. Los datos de impedancias obtenidos se ajustaron al circuito eléctrico equivalente mostrado en la figura 1; donde, $R_{s}$ es la resistencia de la solución, $R_{c t}$ es la resistencia a la transferencia de carga, y $\mathrm{C}_{\mathrm{dl}}$ es la capacitancia de la doble capa eléctrica. Debido a que los semicírculos de las curvas de Nyquist presentaron una depresión, el elemento capacitivo fue reemplazado por un elemento de fase constante (CPE) para obtener una mayor precisión en el ajuste de los datos [9]. Con este circuito equivalente, el ajuste se realizó utilizando el software EIS3000.

Las eficiencias de inhibición del ML se calcularon a partir de valores de $\mathrm{R}_{\mathrm{ct}}$ obtenidos desde los ensayos de impedancia, usando la siguiente ecuación:

$$
E I(\%)=100\left[\frac{R_{c t(i n h)}-R_{c t}}{R_{c t(i n h)}}\right]
$$


donde $\mathrm{R}_{\mathrm{ct}} \mathrm{y} \mathrm{R}_{\mathrm{ct} \text { (inh) }}$ son los valores de resistencia a la transferencia de carga sin y con inhibidor, respectivamente. Para el cálculo de la densidad de corriente de corrosión $\left(\mathrm{A} / \mathrm{cm}^{2}\right)$ se utilizó la ecuación (4) reemplazando $R_{p}$ por $R_{c t}$. Las pendientes de Tafel anódica y catódica (V/década) utilizadas fueron las obtenidas desde los ensayos de modulación de frecuencia electroquímica. La $\mathrm{V}_{\text {corr }}$ en (mm/año) se obtuvo mediante la ecuación (2).

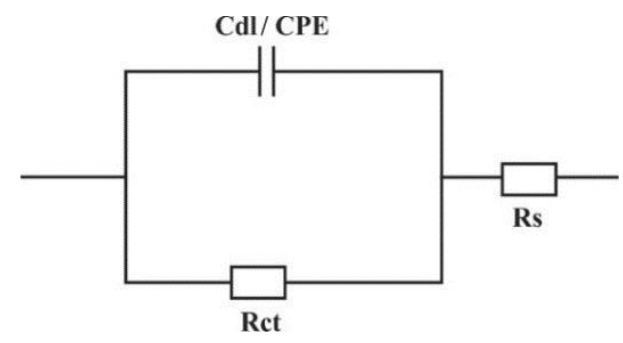

Figura 1: Circuito equivalente usado para ajustar los datos experimentales de impedancia obtenidos con el acero A192 en soluciones de $\mathrm{HCl} 0,5 \mathrm{M}$.

\subsection{Análisis superficial}

La morfología de la superficie de las muestras de acero, expuestas a diferentes soluciones de ensayo, se evaluó mediante microscopía electrónica de barrido (microscopio Tescan, modelo Vega-3). Las muestras del acero con medidas de $30 \mathrm{~mm}$ x $20 \mathrm{~mm}$ x $5 \mathrm{~mm}$ se desbastaron sucesivamente con papeles de carburo de silicio de diferentes grados (220 \# - 2000 \#) y posteriormente se pulieron usando paños impregnados con pasta de diamante de 5 y $1 \mu \mathrm{m}$. Las muestras limpias se sumergieron durante 4 h y 24 h en la solución de $\mathrm{HCl} 0,5$ M sin y con 1,0 g/l de ML a $(298 \pm 1) \mathrm{K}$, que corresponde a la concentración de ML donde se obtuvo la máxima eficiencia de inhibición de la corrosión; luego se lavaron con agua destilada, se secaron en aire caliente y se sometieron a examen de la superficie.

\section{RESULTADOS Y DISCUSIÓN}

\subsection{Caracterización del mucílago de Linum}

El espectro del ML exhibió todas las bandas típicas y picos característicos de los polisacáridos (Figura 2). Las absorciones en el rango de número de onda de 950-1150 $\mathrm{cm}^{-1}$ son atribuidos a uniones glicosídico (C-OC). La absorción a números de onda $1039 \mathrm{~cm}^{-1}$ le corresponde a uniones de los polisacáridos galactosa, arabinosa y ramosa [10]. La absorción a números de onda $1417 \mathrm{~cm}^{-1}$ es causada por el estiramiento simétrico C$\mathrm{OO}$, mientras que la absorción en el número de onda $1600 \mathrm{~cm}^{-1}$ es debido al estiramiento asimétrico C-OO. La absorción en el pico alrededor de $1740 \mathrm{~cm}^{-1}$ es debido a las vibraciones de estiramiento $\mathrm{C}=\mathrm{O}$, que podría deberse a la presencia de aminoácidos (proteínas) [11]. La absorción en el número de onda de $2929 \mathrm{~cm}^{-1} \mathrm{se}$ refiere a la absorción $\mathrm{C}-\mathrm{H}$; estos incluyen $\mathrm{CH}, \mathrm{CH}_{2}$ y $\mathrm{CH}_{3}$ estiramiento y vibraciones de flexión, simétrica, y asimétrica y ocasionalmente dobles solapamientos con $\mathrm{O}-\mathrm{H}$; mientras que la absorción en el número de onda a $3340 \mathrm{~cm}^{-1}$ se debe a las vibraciones de estiramiento O-H [12]. Por lo tanto, se puede entender que el ML es un hidrocoloide que tiene una pequeña cantidad de aminoácidos en su compuesto. Además, los espectros FTIR del ML muestran la presencia de grupos carboxilo, que pueden servir como sitios de unión para los iones.

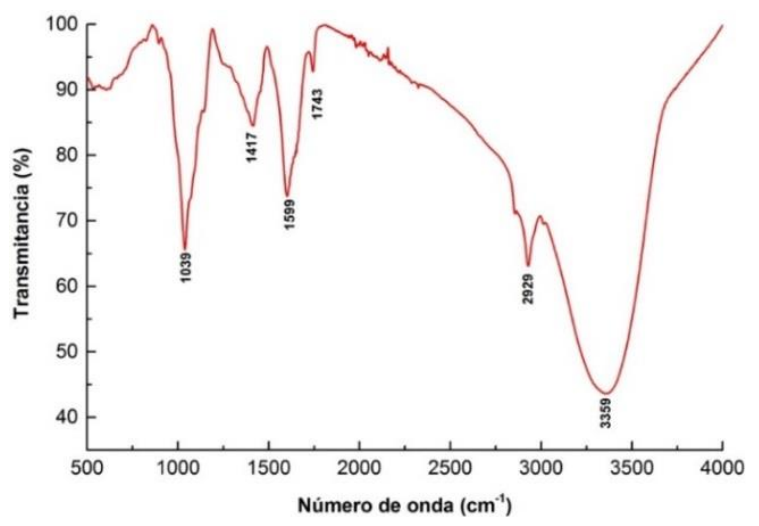

Figura 2: Espectro FT-IR del mucilago de Linum usitatissimun 


\subsection{Polarización potenciodinámica}

La figura 3 muestra el efecto de la concentración del ML en las curvas de polarización potenciodinámicas del acero A192 en $\mathrm{HCl} 0,5 \mathrm{M}$, a $25^{\circ} \mathrm{C}, 45^{\circ} \mathrm{C}$ y $65^{\circ} \mathrm{C}$. Con el aumento de la concentración del ML, se observa el desplazamiento de las curvas anódicas y catódicas a valores menores de densidades de corriente, es decir, provoca una disminución en la velocidad de corrosión ( $\mathrm{i}_{\text {corr }}$ ). Asimismo, el aumento de la concentración del ML produce un desplazamiento del $\mathrm{E}_{\text {corr }}$ hacia potenciales más nobles para las temperaturas de $25{ }^{\circ} \mathrm{C}$ y $45^{\circ} \mathrm{C}$ y un desplazamiento a potenciales más activos a $65^{\circ} \mathrm{C}$. Este cambio indica que a bajas temperaturas las moléculas del ML son más adsorbidas en los sitios anódicos, inhibiendo las reacciones anódicas. Generalmente, si el desplazamiento del $\mathrm{E}_{\text {corr }}$ es mayor a $85 \mathrm{mV}$ con respecto a $\mathrm{E}_{\text {corr }}$ de la solución desinhibida, el inhibidor puede ser considerado como de tipo catódico o anódico [13]. En este estudio los desplazamientos máximos fueron de $81 \mathrm{mV}\left(25^{\circ} \mathrm{C}\right), 42 \mathrm{mV}\left(45^{\circ} \mathrm{C}\right)$ y $-12 \mathrm{mV}\left(65^{\circ} \mathrm{C}\right)$, lo que indica que conforme incrementa la temperatura el comportamiento del ML pasa desde un inhibidor de tipo anódico a uno de tipo mixto.
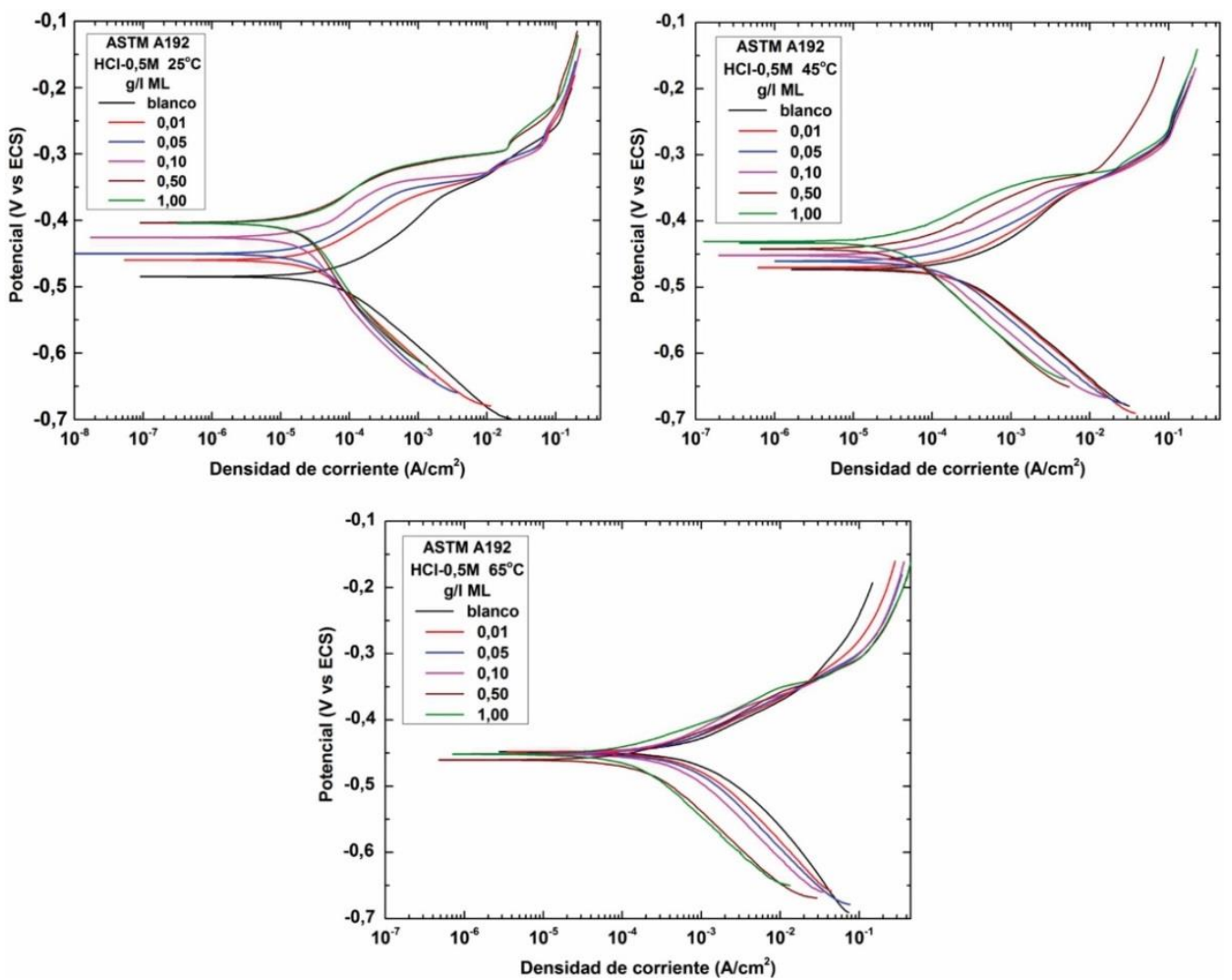

Figura 3: Curvas de polarización del acero ASTM A192 en $\mathrm{HCl}$ 0,5M conteniendo diferentes concentraciones de mucílago de linum $(\mathrm{g} / \mathrm{l})$ a $25^{\circ} \mathrm{C}, 45^{\circ} \mathrm{C}$ y $65^{\circ} \mathrm{C}$.

El efecto de la temperatura sobre la reacción metal/ácido-inhibido puede producir diversos cambios en la superficie del metal, tales como la desorción, descomposición o reordenamiento del inhibidor en la superficie del metal afectando su efecto inhibidor. Sin embargo, según la figura 3, el efecto inhibidor del ML no se ve afectado por una temperatura de $65^{\circ} \mathrm{C}$.

La técnica de extrapolación Tafel utilizada para la determinación de la densidad de corriente de corrosión ( $\mathrm{i}_{\text {corr }}$ ) a partir de las curvas potenciodinámicas, presenta ciertos inconvenientes en su aplicación [14]. Esta técnica fue desarrollada para procesos electroquímicos que presentan las siguientes condiciones: (a) reacciones anódica y catódica controladas solo por transferencia de carga, (b) no existe caída óhmica por presencia de películas superficiales, (c) no existe control mixto (difusión + transferencia de carga), (d) no existe reacciones electroquímicas secundarias, y (e) no se producen cambios significativos en el metal por las fuertes polarizaciones anódicas y catódicas. Las curvas potenciodinámicas obtenidas presentaron cierta curvatura en el rango de potencial a ser evaluado, probablemente generados por un control mixto en la rama catódica y la presencia de óxido en la rama anódica; esto no permitió una determinación precisa de las pen- 
dientes de Tafel anódica y catódica, como tampoco de la densidad de corriente de corrosión, lo que produce una determinación imprecisa del efecto inhibidor del ML, por lo que estos valores fueron obtenidos con la técnica de modulación de frecuencia electroquímica. Sin embargo, un análisis cualitativo a partir de la figura 3 muestra que conforme se incrementa la dosificación del ML, a las temperaturas evaluadas, se produce una disminución considerable en la densidad de corriente anódica y catódica, producto de la adsorción de los compuestos orgánicos presentes en el ML sobre los sitios activos de la superficie del acero, retardando tanto su disolución como la reacción de evolución del hidrógeno, y consecuentemente disminuyendo el proceso corrosivo. Asimismo, el efecto inhibidor del ML es sustancial aun a altas temperaturas.

\subsection{Modulación de frecuencia electroquímica}

La modulación de frecuencia electroquímica (EFM) es una técnica no destructiva que permite la determinación directa tanto de la densidad de corriente de corrosión como de las pendientes de Tafel [8]. La figura 4 muestra los espectros de intermodulación para el acero A192 en soluciones de $\mathrm{HCl}$ 0,5 M sin y con diferentes concentraciones $(0,01$ a $1,0 \mathrm{~g} / \mathrm{l})$ del $\mathrm{ML}$ a diferentes temperaturas, y en la tabla 2 los parámetros electroquímicos obtenidos del proceso corrosivo.

El análisis de la tabla 2, indica que: (a) la velocidad de corrosión del acero A192 en $\mathrm{HCl}$ 0,5M (sin inhibidor) incrementa con la temperatura; (b) un incremento en la concentración del ML disminuye la velocidad de corrosión, para todas las temperaturas evaluadas; (c) el efecto inhibidor del ML (\%EI) incrementa con la temperatura; y (d) los factores de causalidad CF2 y CF3 obtenidos bajo las diferentes condiciones experimentales evaluadas son próximos a los valores teóricos de 2 y 3 , indicando que los datos de densidades de corriente corrosión y las pendientes de Tafel obtenidos por la técnica EFM son confiables.
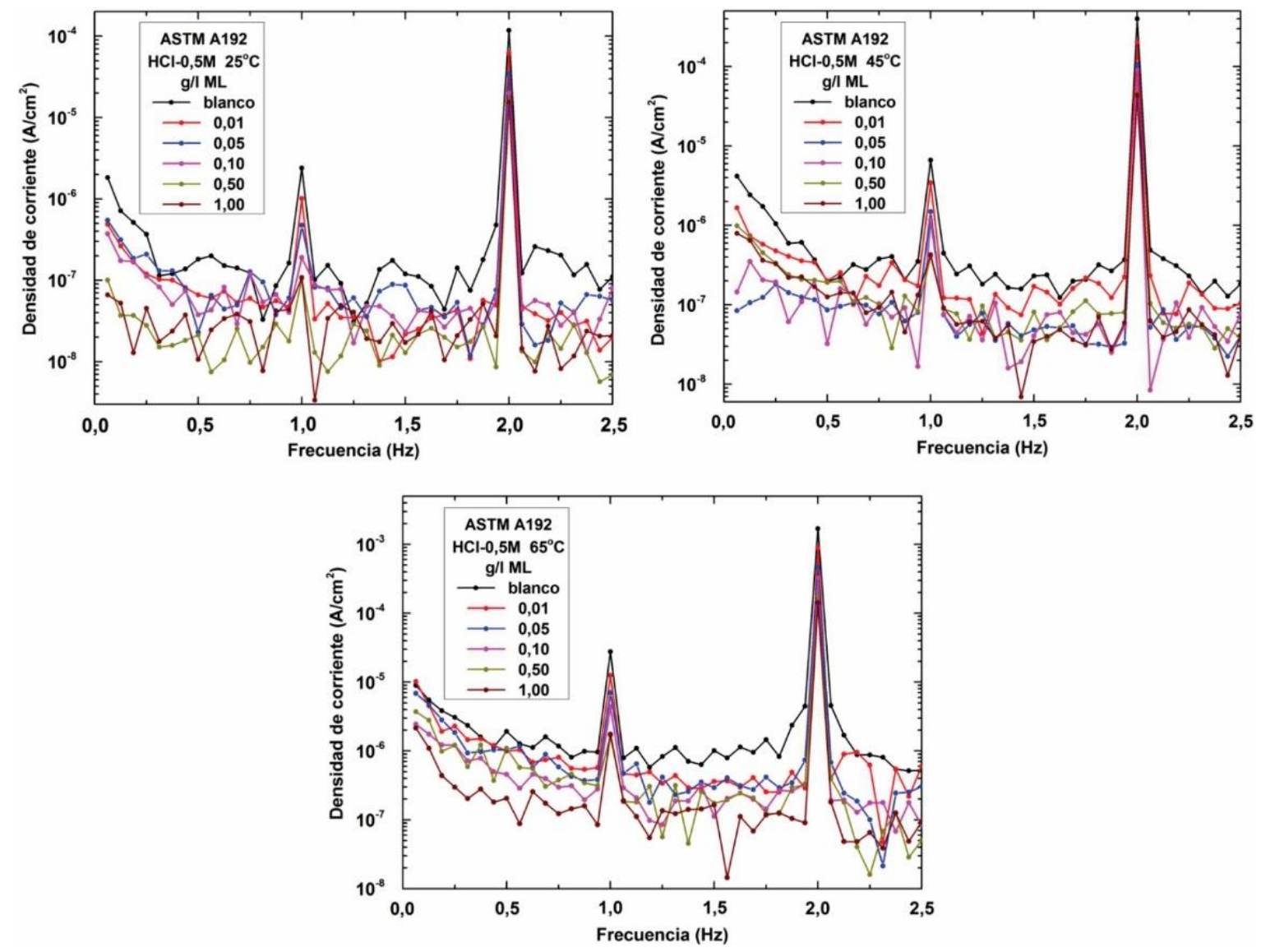

Figura 4: Efecto de la dosificación del ML en la solución de $\mathrm{HCl}$ 0,5M sobre los espectros de intermodulación del acero ASTM A192, a diferentes temperaturas. 
Tabla 2: Parámetros electroquímicos obtenidos mediante la técnica EFM para el acero ASTM A192 en una solución de $\mathrm{HCl}$ 0,5 M sin y con diferentes concentraciones del ML a diferentes temperaturas.

\begin{tabular}{|c|c|c|c|c|c|c|c|c|}
\hline $\begin{array}{c}\mathrm{T} \\
\left({ }^{\circ} \mathrm{C}\right) \\
\end{array}$ & $\begin{array}{l}\mathrm{ML} \\
(\mathrm{g} / \mathrm{l})\end{array}$ & $\begin{array}{c}\mathbf{i}_{\text {corr (EFM) }} \\
\left(\mu \mathrm{A} / \mathrm{cm}^{2}\right)\end{array}$ & $\begin{array}{c}\beta_{\mathrm{a}} \\
\text { (mV/década) }\end{array}$ & $\begin{array}{c}-\beta_{\mathrm{c}} \\
(\mathrm{mV} / \text { década })\end{array}$ & $\begin{array}{c}V_{\text {corr }} \\
\text { (mm/año) }\end{array}$ & $\begin{array}{c}\mathrm{El}_{\mathrm{EFM}} \\
(\%) \\
\end{array}$ & CF (2) & CF (3) \\
\hline \multirow[b]{6}{*}{25} & 0,00 & 150,78 & 84,09 & 89,91 & 1,751 & 0,00 & 2,352 & 3,089 \\
\hline & 0,01 & 98,21 & 91,33 & 99,03 & 1,140 & 34,86 & 2,067 & 3,359 \\
\hline & 0,05 & 61,69 & 105,08 & 116,08 & 0,716 & 59,09 & 2,124 & 2,976 \\
\hline & 0,10 & 40,16 & 106,43 & 141,70 & 0,466 & 73,37 & 2,032 & 3,226 \\
\hline & 0,50 & 35,33 & 112,56 & 134,84 & 0,410 & 76,57 & 2,003 & 2,890 \\
\hline & 1,00 & 34,54 & 129,27 & 166,70 & 0,401 & 77,09 & 1,882 & 2,894 \\
\hline \multirow[b]{6}{*}{45} & 0,00 & 614,22 & 86,47 & 103,00 & 7,131 & 0,00 & 1,990 & 2,922 \\
\hline & 0,01 & 298,47 & 84,70 & 101,72 & 3,465 & 51,34 & 1,937 & 3,035 \\
\hline & 0,05 & 176,80 & 93,17 & 110,63 & 2,053 & 71,21 & 1,931 & 3,243 \\
\hline & 0,10 & 155,93 & 92,35 & 134,75 & 1,810 & 74,62 & 1,926 & 2,930 \\
\hline & 0,50 & 95,88 & 119,50 & 153,00 & 1,113 & 84,39 & 1,953 & 2,985 \\
\hline & 1,00 & 84,69 & 110,50 & 137,00 & 0,983 & 86,22 & 1,954 & 3,161 \\
\hline \multirow[b]{6}{*}{65} & 0,00 & 2833,94 & 85,67 & 110,53 & 32,902 & 0,00 & 2,021 & 3,162 \\
\hline & 0,01 & 1388,51 & 92,08 & 113,20 & 16,121 & 51,01 & 1,982 & 3,088 \\
\hline & 0,05 & 691,90 & 86,38 & 111,15 & 8,033 & 75,59 & 1,929 & 3,077 \\
\hline & 0,10 & 511,26 & 89,89 & 132,20 & 5,936 & 81,96 & 1,964 & 2,988 \\
\hline & 0,50 & 341,77 & 97,79 & 167,85 & 3,968 & 87,94 & 1,964 & 3,281 \\
\hline & 1,00 & 251,02 & 92,72 & 143,50 & 2,914 & 91,14 & 1,971 & 2,968 \\
\hline
\end{tabular}

\subsection{Resistencia a la polarización lineal}

La tabla 3 muestra los valores electroquímicos obtenidos mediante esta técnica sin y con diferentes concentraciones del ML a diferentes temperaturas. Para el cálculo de la densidad de corriente de corrosión se utilizó las pendientes de Tafel anódica y catódica obtenidas con la técnica EFM, según la ecuación 4. El cálculo de la $\mathrm{V}_{\text {corr }}$, se realizó según la ecuación (2). Dos aspectos importantes se observa en la tabla 3. En primer lugar, el incremento en la concentración del ML produce un aumento en la resistencia a la polarización para todas las temperaturas evaluadas; y en segundo lugar, el efecto inhibidor del ML (\%EI) incrementa con la temperatura. Los valores de velocidad de corrosión y eficiencia de inhibición obtenidos por esta técnica electroquímica están en concordancia con los obtenidos a partir de la técnica EFM.

Tabla 3: Parámetros electroquímicos obtenidos mediante la técnica de resistencia a la polarización lineal para el acero ASTM A192 en una solución de $\mathrm{HCl}$ 0,5 M sin y con diferentes concentraciones del ML a diferentes temperaturas.

\begin{tabular}{|c|c|c|c|c|c|}
\hline $\begin{array}{c}\mathrm{T} \\
\left({ }^{\circ} \mathrm{C}\right)\end{array}$ & $\begin{array}{l}M L \\
(g / l)\end{array}$ & $\begin{array}{c}\mathbf{R}_{\mathrm{p}} \\
\left(\Omega . \mathrm{cm}^{2}\right)\end{array}$ & $\begin{array}{c}\mathbf{i}_{\mathrm{corr}(\mathrm{Rp})} \\
\left(\mathrm{mA} / \mathrm{cm}^{2}\right)\end{array}$ & $\begin{array}{c}\mathrm{V}_{\text {corr (Rp) }} \\
(\mathrm{mm} / \mathbf{a n ̃ o})\end{array}$ & $\begin{array}{l}\mathrm{El}_{\mathrm{Rp}} \\
(\%)\end{array}$ \\
\hline \multirow[t]{6}{*}{25} & 0,00 & 131,12 & 144,68 & 1,680 & \\
\hline & 0,01 & 223,23 & 92,48 & 1,074 & 41,26 \\
\hline & 0,05 & 472,05 & 51,14 & 0,594 & 72,22 \\
\hline & 0,10 & 686,77 & 38,57 & 0,448 & 80,91 \\
\hline & 0,50 & 888,33 & 30,14 & 0,350 & 85,24 \\
\hline & 1,00 & 1116,69 & 28,35 & 0,329 & 88,26 \\
\hline \multirow[t]{5}{*}{45} & 0,00 & 32,02 & 646,66 & 7,508 & \\
\hline & 0,01 & 64,44 & 311,47 & 3,616 & 50,32 \\
\hline & 0,05 & 123,39 & 178,17 & 2,068 & 74,05 \\
\hline & 0,10 & 146,83 & 162,59 & 1,888 & 78,19 \\
\hline & 0,50 & 295,56 & 99,25 & 1,152 & 89,17 \\
\hline
\end{tabular}




\begin{tabular}{r|r|r|r|r|r}
\hline & 1,00 & 333,76 & 79,59 & 0,924 & 90,41 \\
\hline \multirow{6}{*}{65} & 0,00 & 7,45 & 2825,34 & 32,802 & \\
\cline { 2 - 6 } & 0,01 & 14,08 & 1565,59 & 18,176 & 47,13 \\
\cline { 2 - 6 } & 0,05 & 29,86 & 707,83 & 8,218 & 75,06 \\
\hline & 0,10 & 41,56 & 561,12 & 6,515 & 82,08 \\
\cline { 2 - 6 } & 0,50 & 79,46 & 337,68 & 3,920 & 90,63 \\
\cline { 2 - 7 } & 1,00 & 93,31 & 363,97 & 3,065 & 92,02 \\
\hline
\end{tabular}

\subsection{Espectroscopia de impedancia electroquímica}

Los diagramas de impedancia electroquímica para el acero A192 en una solución de $\mathrm{HCl} 0,5$ a $25^{\circ} \mathrm{C}, 45^{\circ} \mathrm{C}$ y $65^{\circ} \mathrm{C}$, en ausencia y presencia de varias concentraciones de ML, son mostrados en la figura 5. Asimismo, los resultados promedios de los datos de impedancia obtenidos en estos ensayos son resumidos en la tabla 4 . Similar a los ensayos de resistencia a la polarización, para el cálculo de la densidad de corriente de corrosión se utilizó las pendientes de Tafel anódica y catódica obtenidas con la técnica EFM.

Los diagramas de impedancia electroquímica, en ausencia y presencia del ML a diferentes temperaturas, muestran un único lazo capacitivo deprimido, lo que se le atribuye a un proceso corrosivo controlado por activación. Haciendo referencia a la tabla 4, con el incremento de la concentración del ML se observa dos aspectos importantes: (a) la resistencia de transferencia de carga $\left(\mathrm{R}_{\mathrm{ct}}\right)$ aumenta significativamente, y (b) una disminución en el valor de la capacitancia $\left(\mathrm{C}_{\mathrm{dl}}\right)$, que puede ser causada por la reducción de la constante dieléctrica local y/o por un aumento en el espesor de la doble capa eléctrica. Estos resultados muestran que la presencia del mucílago modifica la estructura de la doble capa eléctrica, lo que sugiere que las moléculas inhibidoras actúan por adsorción en la interfase metal/solución. La resistencia de la solución $\left(\mathrm{R}_{\mathrm{s}}\right)$ no se ve afectada marcadamente en presencia del ML

Asimismo, el incremento de la resistencia de transferencia de carga y la disminución de la capacitancia, con el aumento de la concentración del ML, indica una disminución en el área superficial activa causada por la adsorción de los inhibidores sobre la superficie de acero, y sugiere que el proceso de corrosión se vio obstaculizado; esta hipótesis es corroborada mediante las curvas de polarización anódica y catódica y los potencial de corrosión.

Los mejores resultados de eficiencia de inhibición de la corrosión fueron del $87,6 \%\left(25^{\circ} \mathrm{C}\right), 90,3 \%$ $\left(45^{\circ} \mathrm{C}\right)$ y $92,2 \%\left(65^{\circ} \mathrm{C}\right)$, obtenidos con $1,0 \mathrm{~g} / 1$ del $\mathrm{ML}$; no obstante con $0,5 \mathrm{~g} / \mathrm{l}$ del $\mathrm{ML}$, las eficiencias de inhibición fueron óptimas. Otro aspecto importante a remarcar fue que el efecto inhibidor del ML incrementa con la temperatura, como se observa en la tabla 4. 

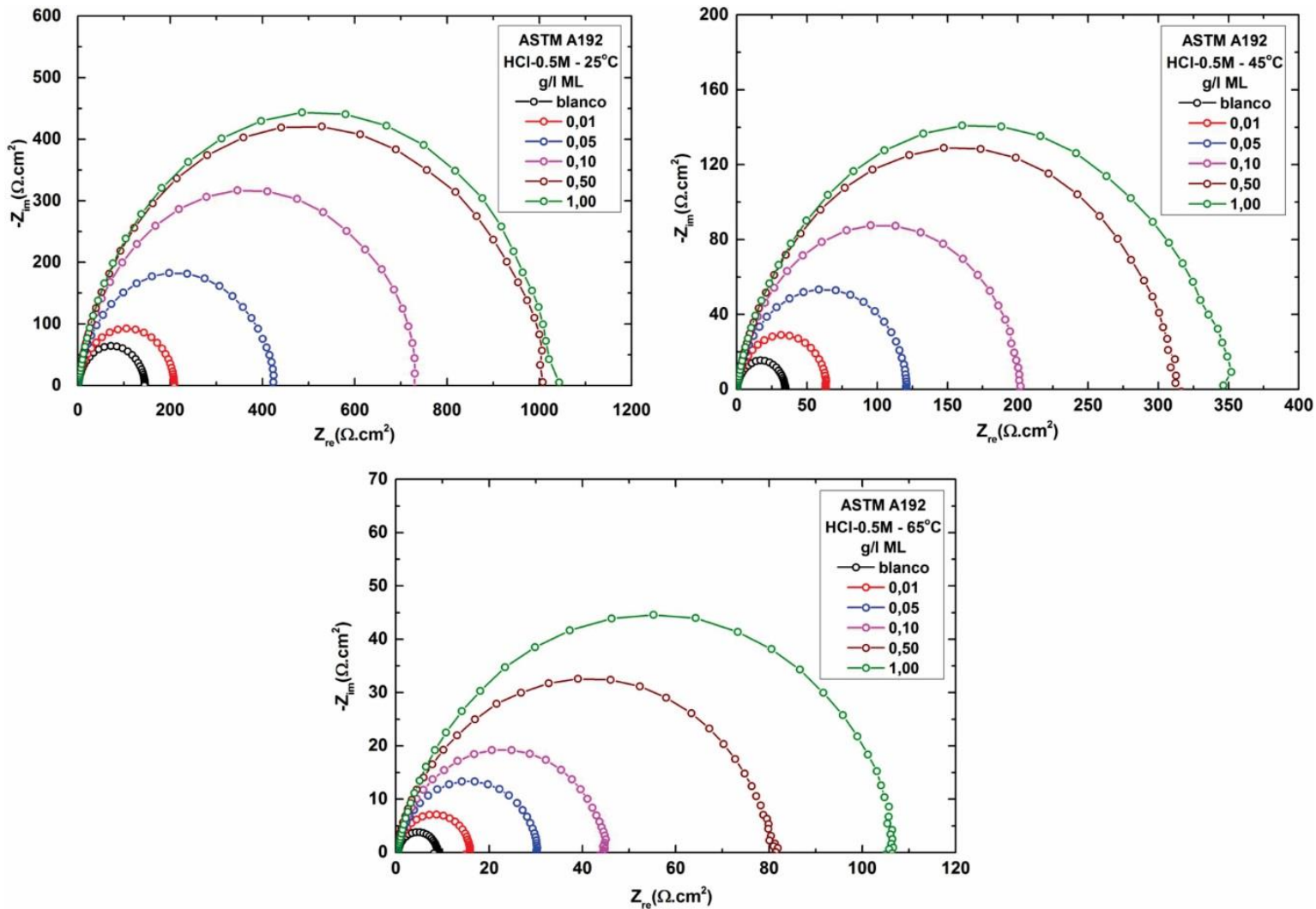

Figura 5: Diagrama de Nyquist obtenidos al potencial de corrosión del acero ASTM A192 en la solución de HCl 0,5M, en ausencia y presencia del ML, a diferentes temperaturas.

Tabla 4: Parámetros electroquímicos obtenidos mediante la técnica EIS para el acero ASTM A192 en una solución de $\mathrm{HCl}$ 0,5 M sin y con diferentes concentraciones del ML a diferentes temperaturas.

\begin{tabular}{|c|c|c|c|c|c|c|c|c|}
\hline $\begin{array}{c}\mathrm{T} \\
\left({ }^{\circ} \mathrm{C}\right) \\
\end{array}$ & $\begin{array}{l}M L \\
(g / l)\end{array}$ & $\begin{array}{c}\mathbf{R}_{\mathrm{s}} \\
\left(\Omega . \mathrm{cm}^{2}\right) \\
\end{array}$ & $\begin{array}{c}\mathbf{R}_{\mathrm{ct}} \\
\left(\Omega \cdot \mathrm{cm}^{2}\right)\end{array}$ & $\mathbf{n}$ & $\begin{array}{c}C_{d l} \\
\left(\mu F / \mathrm{cm}^{2}\right)\end{array}$ & $\begin{array}{c}\mathbf{i}_{\text {corr }(E I S)} \\
\left(\mu \mathbf{A} / \mathbf{c m}^{2}\right)\end{array}$ & $\begin{array}{c}\mathrm{V}_{\text {corr (EIS) }} \\
\text { (mm/año) }\end{array}$ & $\begin{array}{c}\mathrm{EI}_{\mathrm{EIS}} \\
(\%)\end{array}$ \\
\hline \multirow[t]{6}{*}{25} & 0,00 & 0,81 & 134,10 & 0,944 & 87,25 & 141,43 & 1,642 & \\
\hline & 0,01 & 0,56 & 225,80 & 0,940 & 87,08 & 91,37 & 1,061 & 4,61 \\
\hline & 0,05 & 0,48 & 438,47 & 0,923 & 66,44 & 54,98 & 0,638 & 69,42 \\
\hline & 0,10 & 0,38 & 697,80 & 0,917 & 63,09 & 37,90 & 0,440 & 80,78 \\
\hline & 0,50 & 0,38 & 893,26 & 0,904 & 58,12 & 29,94 & 0,348 & 84,99 \\
\hline & 1,00 & 0,44 & 1077,98 & 0,904 & 51,92 & 29,36 & 0.341 & 87,56 \\
\hline \multirow[t]{6}{*}{45} & 0,00 & 0,50 & 32,50 & 0,953 & 125,23 & 629,40 & 7,307 & \\
\hline & 0,01 & 0,26 & 63,78 & 0,964 & 120,15 & 314,76 & 3,654 & 49,05 \\
\hline & 0,05 & 0,39 & 119,66 & 0,930 & 83,41 & 183,34 & 2,128 & 72,84 \\
\hline & 0,10 & 0,36 & 141,88 & 0,932 & 81,58 & 168,46 & 1,956 & 77,10 \\
\hline & 0,50 & 0,32 & 318,41 & 0,912 & 49,89 & 91,55 & 1,063 & 89,79 \\
\hline & 1,00 & 0,39 & 336,18 & 0,906 & 46,52 & 79,01 & 0,917 & 90,33 \\
\hline \multirow[t]{6}{*}{65} & 0,00 & 0,26 & 7,68 & 0,995 & 288,12 & 2794,86 & 32,45 & \\
\hline & 0,01 & 0,34 & 15,27 & 0,968 & 190,12 & 1443,86 & 16,76 & 49,71 \\
\hline & 0,05 & 0,37 & 29,98 & 0,942 & 147,31 & 704,40 & 8,18 & 74,38 \\
\hline & 0,10 & 0,36 & 43,44 & 0,931 & 120,59 & 535,03 & 6,21 & 82,32 \\
\hline & 0,50 & 0,30 & 78,40 & 0,913 & 57,66 & 342,22 & 3,97 & 90,20 \\
\hline & 1,00 & 0,35 & 98,18 & 0,918 & 56,75 & 252,22 & 2,93 & 92,18 \\
\hline
\end{tabular}




\subsection{Isoterma de adsorción del mucílago de Linum en el acero ASTM A192}

El mecanismo de acción de los inhibidores en solución ácida es generalmente por su adsorción en la superficie del metal. BOCKRIS y SWINKELS [15], establecieron que la adsorción de una sustancia orgánica sobre la superficie del metal puede ser expresada mediante la siguiente reacción:

$$
M\left(n \mathrm{H}_{2} \mathrm{O}\right)_{a d s}+I_{(\mathrm{sol})} \leftrightarrow M I_{a d s}+n\left(\mathrm{H}_{2} \mathrm{O}\right)_{\text {sol }}
$$

donde $\mathrm{n}$ es el número de moléculas de agua desplazadas de la superficie del metal por una molécula del inhibidor adsorbido; el valor de $\mathrm{n}$ depende de la sección transversal de la molécula orgánica con respecto a la molécula del agua.

La adsorción de las moléculas orgánicas ocurre debido a que la energía de interacción entre el inhibidor y la superficie del metal es más fuerte que la existente entre la molécula de agua y la superficie del metal [15]. La adsorción en las superficies corroídas nunca alcanza el equilibrio real y tiende a alcanzar un estado estacionario de adsorción. Sin embargo, cuando la velocidad de corrosión es suficientemente pequeña, el estado estacionario de adsorción tiene una tendencia a convertirse en un estado de cuasi-equilibrio. En este caso, es razonable considerar la adsorción de cuasi-equilibrio desde un punto de vista termodinámico utilizando las isotermas de adsorción de equilibrio apropiadas [16].

Cuando se alcanza el cuasi-equilibrio en el proceso de adsorción del ML en la superficie del metal, es posible obtener diversas expresiones de gráficos de isotermas de adsorción. El grado de protección de la superficie $(\theta=\mathrm{IE}(\%) / 100)$, para las diferentes técnicas utilizadas, puede ser obtenido en función de la concentración del ML (inhibidor), como se muestra en la tabla 5.

Tabla 5: Valores del grado de protección obtenido desde las técnicas de EFM, Rp y EIS para el acero ASTM A192 en una solución de $\mathrm{HCl}$ 0,5 M, con diferentes concentraciones del ML a diferentes temperaturas.

\begin{tabular}{|c|c|c|c|c|}
\hline \multirow{2}{*}{$\begin{array}{c}\mathrm{T} \\
\left({ }^{\circ} \mathrm{C}\right) \\
\end{array}$} & \multirow{2}{*}{$\begin{array}{c}\text { Mucílago de linaza } \\
(\mathrm{g} / \mathrm{l})\end{array}$} & \multicolumn{3}{|c|}{ Grado de protección $(\theta)$} \\
\hline & & Técnica EFM & Técnica Rp & Técnica EIS \\
\hline \multirow{6}{*}{$25 \div C$} & Blanco & 0,000 & 0,000 & 0,000 \\
\hline & 0,01 & 0,349 & 0,413 & 0,406 \\
\hline & 0,05 & 0,591 & 0,722 & 0,694 \\
\hline & 0,10 & 0,734 & 0,809 & 0,808 \\
\hline & 0,50 & 0,766 & 0,852 & 0,850 \\
\hline & 1,00 & 0,771 & 0,883 & 0,876 \\
\hline \multirow{6}{*}{$45^{\circ} \mathrm{C}$} & Blanco & 0,000 & 0,000 & 0,000 \\
\hline & 0,01 & 0,513 & 0,503 & 0,490 \\
\hline & 0,05 & 0,712 & 0,741 & 0,728 \\
\hline & 0,10 & 0,746 & 0,782 & 0,771 \\
\hline & 0,50 & 0,844 & 0,892 & 0,898 \\
\hline & 1,00 & 0,862 & 0,904 & 0,903 \\
\hline \multirow{6}{*}{$65^{\circ} \mathrm{C}$} & Blanco & 0,000 & 0,000 & 0,000 \\
\hline & 0,01 & 0,510 & 0,471 & 0,497 \\
\hline & 0,05 & 0,756 & 0,751 & 0,744 \\
\hline & 0,10 & 0,820 & 0,821 & 0,823 \\
\hline & 0,50 & 0,879 & 0,906 & 0,902 \\
\hline & 1,00 & 0,911 & 0,920 & 0,922 \\
\hline
\end{tabular}

Los valores de $\theta$ incrementan con el aumento de la concentración del ML, como un resultado de una mayor inhibición de las moléculas orgánicas adsorbidas en la superficie del acero. Asimismo, suponiendo que la adsorción del mucilago ocurre por una monocapa adsorbida e ignorando la interacción lateral entre las moléculas inhibidoras, aplicamos la isoterma de adsorción de Langmuir para investigar el mecanismo de adsorción mediante la siguiente ecuación:

$$
\frac{C_{i n h}}{\theta}=\frac{1}{k} C_{i n h}
$$


donde, $\mathrm{C}_{\text {inh }}$ es la concentración de inhibidor en el volumen total de la solución de ensayo, y k es la constante de equilibrio de adsorción involucrados en la reacción química. En la figura 6 se muestra, para las diferentes técnicas utilizadas, la regresión lineal entre $\mathrm{C}_{\mathrm{inh}} / \theta \mathrm{y} \mathrm{C}_{\mathrm{inh}}$, para las diferentes temperaturas evaluadas, mientras que los resultados del ajuste se exponen en la tabla 6.
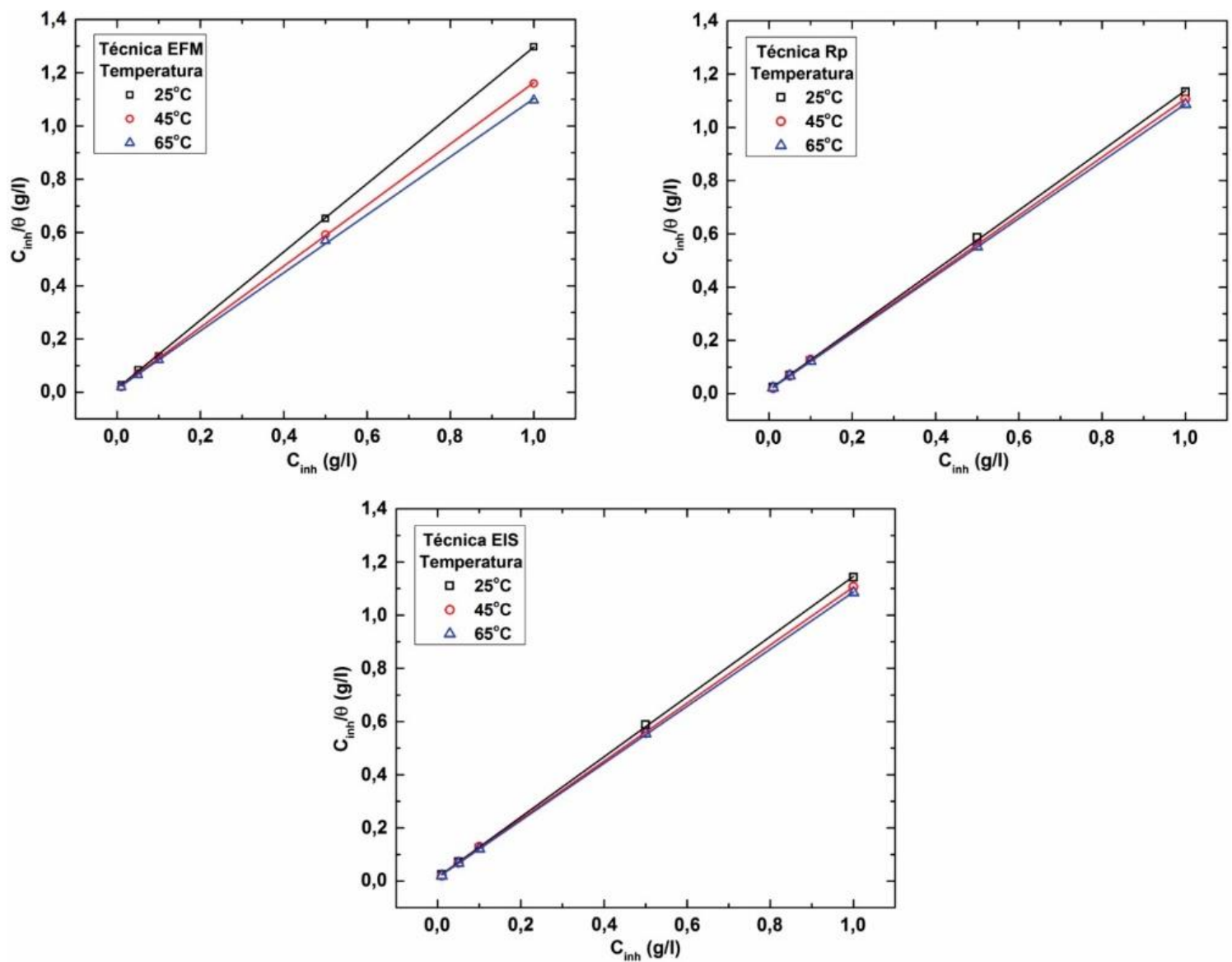

Figura 6: Curva de ajuste de la isoterma de Langmuir para el acero ASTM A192 en HCl 0,5 M para determinar los parámetros termodinámicos de la inhibición de la corrosión del ML a diferentes temperaturas. Técnicas EFM, Rp y EIS.

Los resultados de la tabla 6 , muestran que los coeficientes de correlación lineal fueron muy cercanos a 1,0 dentro del rango de temperatura de 25 a $65{ }^{\circ} \mathrm{C}$, para los resultados de las técnicas EFM, Rp y EIS; asimismo, el valor de la pendiente fue próximo a la unidad. Estos datos indican que la adsorción del ML en la superficie de acero en una solución de $\mathrm{HCl}$ 0,5 M obedece a la isoterma de adsorción de Langmuir. Se observó, en las tres técnicas electroquímicas utilizadas, que la constante de adsorción (k) aumentó al incrementar la temperatura. El valor de $\mathrm{k}$ designa la fuerza de adsorción del inhibidor en la superficie del acero, claramente, el ML dio valores mayores de $\mathrm{k}$ conforme incrementó la temperatura, lo que indica que con el aumento de la temperatura el ML se adsorbe con más fuerza sobre la superficie de acero incrementando su eficacia de inhibición y sugiere que éste es quimisorbido sobre la superficie del acero. 
Tabla 6: Parámetros de la regresión lineal $\mathrm{C}_{\mathrm{inh}} / \theta$ y $\mathrm{C}_{\mathrm{inh}}$, obtenidos desde las técnica de EFM, Rp y EIS.

\begin{tabular}{c|c|c|c|c}
\hline $\begin{array}{c}\text { Temperatura } \\
\left({ }^{\circ} \mathbf{C}\right)\end{array}$ & $\begin{array}{c}\text { Intercepto } \\
(\mathbf{1} / \mathbf{k})\end{array}$ & Pendiente & $\mathbf{k}$ & $\begin{array}{c}\text { Coef. de correlación } \\
\left.\text { lineal } \mathbf{( R}^{2}\right)\end{array}$ \\
\hline Técnica EFM \\
\hline 25 & 0,01461 & 1,281 & 68,44 & 0,9999 \\
\hline 45 & 0,01389 & 1,149 & 71,98 & 0,9999 \\
\hline 65 & 0,01299 & 1,090 & 76,99 & 0,9997 \\
\hline Técnica Rp & & \\
\hline 25 & 0,01430 & 1,124 & 69,91 & 0,9998 \\
\hline 45 & 0,01340 & 1,093 & 74,64 & 0,9999 \\
\hline 65 & 0,01276 & 1,075 & 78,34 & 0,9999 \\
\hline Técnica EIS & & & \\
\hline 25 & 0,01463 & 1,131 & 68,36 & 0,9998 \\
\hline 45 & 0,01393 & 1,092 & 71,77 & 0,9999 \\
\hline 65 & 0,01300 & 1,074 & 76,94 & 0,9999 \\
\hline
\end{tabular}

\subsection{Parámetros termodinámicos de adsorción del mucílago de Linum en el acero ASTM A192}

El efecto inhibidor del ML en la corrosión del acero A192 en $\mathrm{HCl}$ 0,5 M, puede ser evaluado y explicado de una forma más detallada mediante el uso de la termodinámica, así, la entalpia estándar de adsorción $\left(\Delta \mathrm{H}^{\mathrm{o}}{ }_{\mathrm{ads}}\right)$, la energía libre estándar de adsorción $\left(\Delta \mathrm{G}_{\mathrm{ads}}^{\mathrm{o}}\right)$, y la entropía estándar de adsorción $\left(\Delta \mathrm{S}_{\mathrm{ads}}^{\mathrm{o}}\right)$ fueron calculados para dilucidar el fenómeno de la acción inhibidora del ML. Según la ecuación de Van't Hoff [17]:

$$
\ln k=\frac{-\Delta H_{a d s}^{o}}{R T}+\text { const }
$$

para la obtención del calor de adsorción se realizó la regresión lineal entre ln k y 1/T, como se muestra en la figura 7. Bajo las condiciones experimentales, el calor de adsorción podía considerarse aproximadamente como la entalpia estándar de adsorción $\left(\Delta \mathrm{H}^{\mathrm{o}}{ }_{\mathrm{ads}}\right)$, [17]. Para obtener la energía libre estándar de adsorción $\left(\Delta \mathrm{G}^{\mathrm{o}}{ }_{\text {ads }}\right)$, se utilizó la siguiente ecuación [17]:

$$
k=\left(\frac{1}{55,5}\right) \exp \left[\frac{-\Delta G_{a d s}^{o}}{R T}\right]
$$

y considerando la ecuación básica de termodinámica $\Delta \mathrm{G}_{\mathrm{ads}}^{\mathrm{o}}=\Delta \mathrm{H}_{\mathrm{ads}}^{\mathrm{o}}-\mathrm{T} \Delta \mathrm{S}_{\text {ads }}^{\mathrm{o}}$, se obtuvo la entropía estándar de adsorción $\left(\Delta \mathrm{S}_{\text {ads }}^{\mathrm{o}}\right)$. Todos los parámetros termodinámicos obtenidos se muestran en la Tabla 7.

Los valores negativos de $\Delta \mathrm{G}_{\text {ads }}^{\mathrm{o}}$ indican la espontaneidad del proceso de adsorción del inhibidor sobre la superficie del acero. Generalmente, valores de $\Delta \mathrm{G}_{\text {ads }}^{\mathrm{o}}$ menos negativo que - $20 \mathrm{~kJ} / \mathrm{mol}$ significa una interacción electrostática entre las moléculas cargadas y el metal cargado (fisisorción); mientras que valores de $\Delta \mathrm{G}_{\text {ads }}^{\mathrm{o}}$ más negativos que $-40 \mathrm{~kJ} / \mathrm{mol}$ implican el intercambio de carga o de transferencia de electrones desde las moléculas orgánicas hacia la superficie del metal para formar un tipo de enlace coordinado (quimisorción) [18]. METIKOS-HUKOVIC et al. [19], describen la interacción entre la tiourea y el hierro $\left(\Delta \mathrm{G}_{\mathrm{ads}}^{\mathrm{o}}=-39\right.$ $\mathrm{kJ} / \mathrm{mol}$ ) como quimisorción. De manera similar WANG et al., [20], y LI et al. [21], sobre la interacción de acero dulce con mercapto-triazoles $\left(\Delta \mathrm{G}_{\text {ads }}^{\mathrm{o}}=-32.8 \mathrm{~kJ} / \mathrm{mol}\right)$ y o-fenantrolina $\left(\Delta \mathrm{G}_{\mathrm{ads}}^{\mathrm{o}}=-24.8 \mathrm{~kJ} / \mathrm{mol}\right)$ respectivamente, lo describen como quimisorción. Basándose en los datos presentados en la tabla 7, los valores calculados de $\Delta \mathrm{G}_{\text {ads }}^{\mathrm{o}}$ infieren que el mecanismo de adsorción del ML sobre la superficie del acero A192 en soluciones de $\mathrm{HCl}$ 0,5 M es típico de adsorción química. 


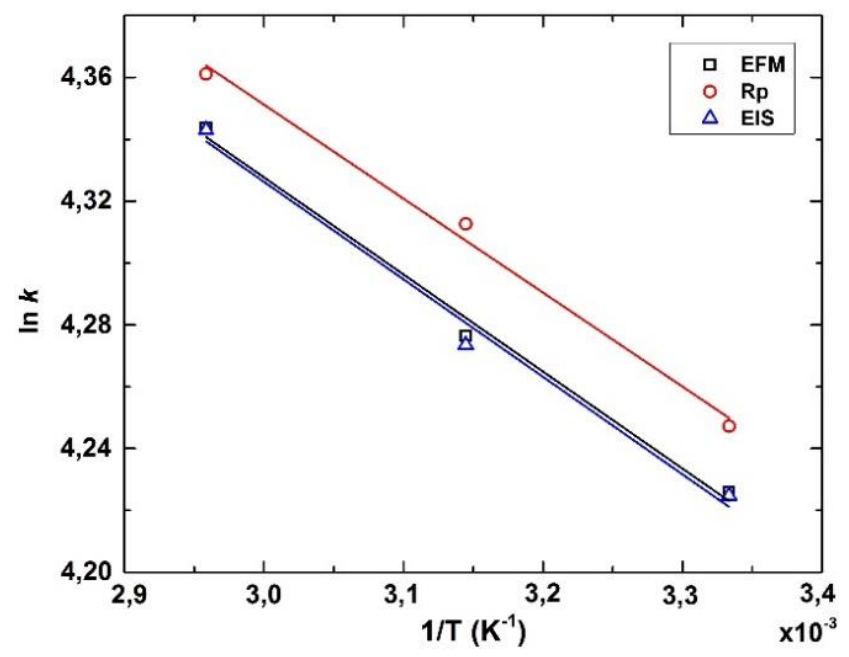

Figura 7: Curva de ajuste de la ecuación de Van't Hoff para determinar la entalpia estándar de adsorción del ML sobre el acero ASTM A192 en HCl 0,5 M. Técnicas EFM, Rp y EIS.

Tabla 7: Parámetros termodinámicos de la adsorción del ML sobre la superficie del acero ASTM A192 a diferentes temperaturas; obtenidos mediante las técnicas EFM, Rp y EIS.

\begin{tabular}{c|c|c|c|c}
\hline \multirow{2}{*}{$\begin{array}{c}\text { Temperatura } \\
\left({ }^{\circ} \mathbf{C}\right)\end{array}$} & \multicolumn{3}{|c|}{ Técnica electroquímica } & \multirow{2}{*}{ Promedio } \\
\cline { 2 - 4 } & $\mathbf{E F M}$ & $\mathbf{R p}$ & EIS & \\
\hline & \multicolumn{4}{|c}{$\Delta \mathbf{G}^{\mathbf{0}}{ }_{\text {ads }}(\mathbf{k J} / \mathbf{m o l})$} \\
\hline 25 & $-27,771$ & $-27,824$ & $-27,768$ & $-27,79$ \\
\hline 45 & $-29,571$ & $-29,667$ & $-29,563$ & $-29,60$ \\
\hline 65 & $-31,620$ & $-31,669$ & $-31,618$ & $-31,64$ \\
\hline & \multicolumn{4}{|c}{$\Delta \mathbf{H}^{\mathbf{o}}$ ads } \\
\hline & $(\mathbf{k J} / \mathbf{m o l})$ \\
\hline 45 & 2,610 & 2,527 & 2,622 & 2,586 \\
\hline 65 & 2,610 & 2,527 & 2,622 & 2,586 \\
\hline & 2,610 & 2,527 & 2,622 & 2,586 \\
\hline 25 & 101,271 & 101,171 & 101,301 & 101,248 \\
\hline 45 & 101,198 & 101,238 & 101,211 & 101,216 \\
\hline 65 & 101,272 & 101,171 & 101,301 & 101,248 \\
\hline
\end{tabular}

Otro parámetros termodinámicos importante para el estudio del mecanismo de inhibición de la corrosión por el ML, es la entalpia de adsorción. Mientras que un proceso de adsorción endotérmica $\left(\Delta \mathrm{H}^{\mathrm{o}}\right.$ ads $\left.>0\right)$ se atribuye de manera inequívoca a quimisorción, un proceso de adsorción exotérmica $\left(\Delta \mathrm{H}^{\mathrm{o}}{ }_{\mathrm{ads}}<0\right)$ puede implicar fisisorción o quimisorción o una mezcla de ambos procesos [22]. El valor promedio calculado por las diferentes técnicas para $\Delta \mathrm{H}_{\text {ads }}^{\mathrm{o}}$ es $2,59 \mathrm{~kJ} / \mathrm{mol}$, lo que indica que el ML es químicamente adsorbido sobre la superficie del acero mediante un proceso endotérmico y su adsorción se ve facilitado con el incremento de la temperatura.

\subsection{Parámetros cinéticos de adsorción del mucílago de Linum en el acero ASTM A192}

La temperatura aumenta la velocidad de los procesos electroquímicos y por lo tanto, influencia en la cinética y equilibrio de adsorción del inhibidor. Investigaciones con la variable temperatura permiten determinar la energía de activación y el factor pre-exponencial en ausencia y en presencia del inhibidor. Los resultados obtenidos pueden ayudar a dilucidar el mecanismo de inhibición de la corrosión. Los parámetros de activación para el proceso de corrosión en ausencia y en presencia del ML se calcularon a partir de una gráfica tipo Arrhenius de acuerdo con la siguiente ecuación: 


$$
V_{\text {corr }}=\operatorname{Aexp}\left[\frac{-E_{a}^{*}}{R T}\right]
$$

donde $\mathrm{E}_{\mathrm{a}}^{*}$ es la energía de activación aparente; A es la constante pre-exponencial de Arrhenius, que está relacionado con el número de los centros activos en el caso de reacciones químicas heterogéneas; $\mathrm{R}$ es la constante de los gases y $\mathrm{T}$ la temperatura absoluta.

Gráficos de la ecuación de Arrhenius se construyeron a partir de los datos obtenidos con las técnicas electroquímicas EFM, Rp y EIS, para el acero A192 en soluciones de $\mathrm{HCl}$ 0,5 M conteniendo diversas concentraciones del ML en el rango de la temperatura de $25^{\circ} \mathrm{C}$ y $65^{\circ} \mathrm{C}$. La figura 8 muestra las gráficas de la ecuación de Arrhenius obtenidos a partir de estas técnicas y la tabla 8 lista las energías de activación obtenidas a partir de la pendiente de la gráfica del [log $\left.\mathrm{V}_{\text {corr }}\right]$ vs $[1 / \mathrm{T}]$, como una función de la concentración del inhibidor.
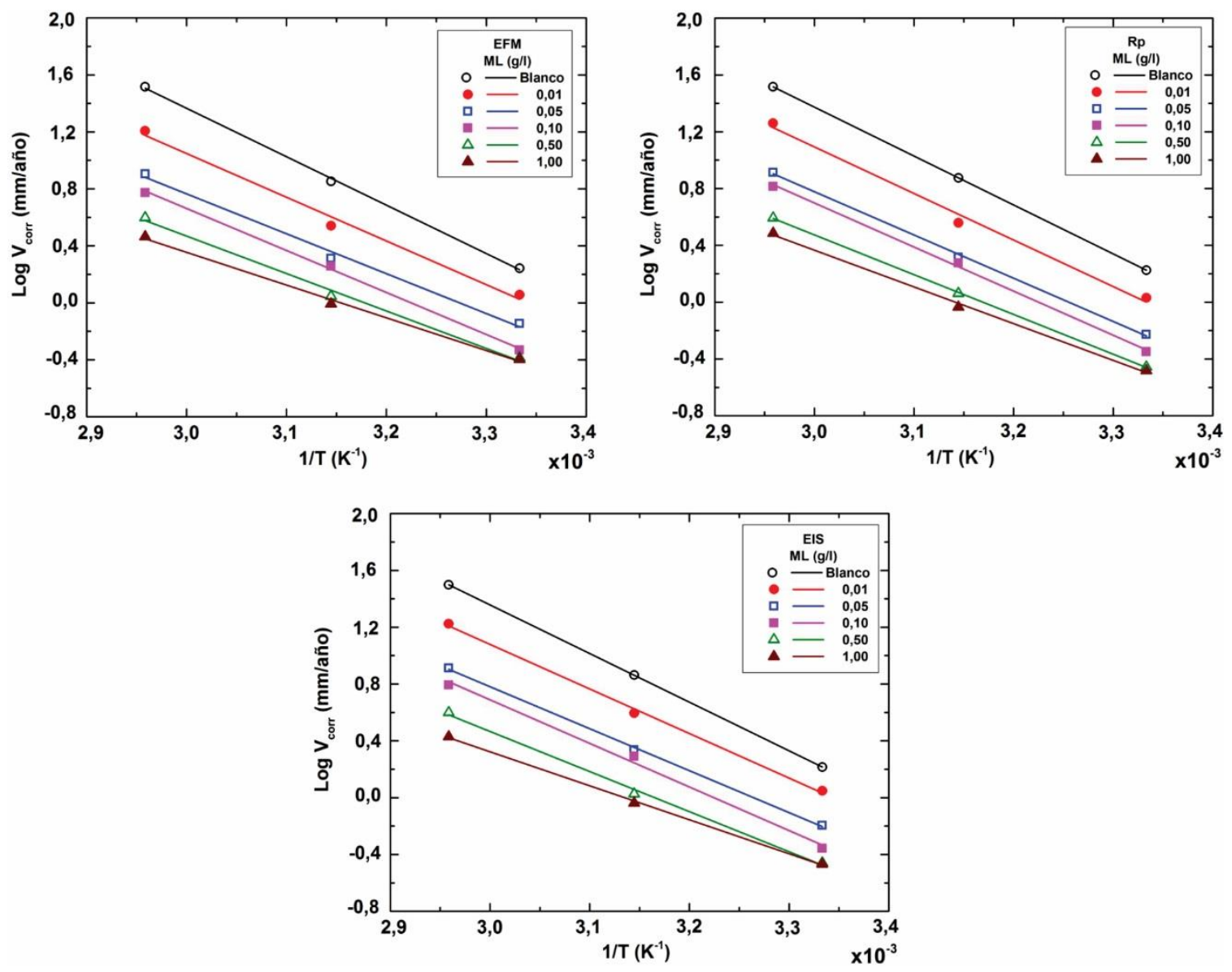

Figura 8: Gráfica de Arrhenius para la velocidad de corrosión del acero ASTM A192 en HCl 0,5 M con diferentes concentraciones del ML. 
Tabla 8: Parámetros obtenidos a partir de la ecuación de Arrhenius.

\begin{tabular}{|c|c|c|c|c|}
\hline $\begin{array}{l}M L \\
(g / l)\end{array}$ & Técnica & $\begin{array}{l}\text { Factor pre-exponencial } \\
\qquad A(\mathrm{~mm} / \mathrm{año})\end{array}$ & $\begin{array}{l}\text { Energía de activación } \\
\qquad E^{\star}{ }_{a}(\mathrm{~kJ} / \mathrm{mol})\end{array}$ & $\begin{array}{l}\text { Coeficiente de } \\
\text { regresión lineal }\end{array}$ \\
\hline \multirow[t]{4}{*}{0,00} & EFM & $3,67 \times 10^{11}$ & 65,10 & 0,9984 \\
\hline & $\mathrm{Rp}$ & $5,08 \times 10^{11}$ & 65,95 & 0,9999 \\
\hline & EIS & $4,26 \times 10^{11}$ & 65,56 & 0,9999 \\
\hline & Promedio & $4,34 \times 10^{11}$ & 65,54 & \\
\hline \multirow[t]{4}{*}{0,01} & EFM & $1,80 \times 10^{10}$ & 58,76 & 0,9817 \\
\hline & $\mathrm{Rp}$ & $8,42 \times 10^{10}$ & 62,75 & 0,9854 \\
\hline & EIS & $3,11 \times 10^{10}$ & 60,08 & 0,9962 \\
\hline & Promedio & $4,44 \times 10^{10}$ & 60,53 & \\
\hline \multirow[t]{4}{*}{0,05} & EFM & $1,47 \times 10^{9}$ & 53,63 & 0,9878 \\
\hline & $\mathrm{Rp}$ & $8,16 \times 10^{9}$ & 58,30 & 0,9978 \\
\hline & EIS & $4,43 \times 10^{9}$ & 56,59 & 0,9985 \\
\hline & Promedio & $4,69 \times 10^{9}$ & 56,17 & \\
\hline \multirow[t]{4}{*}{0,10} & EFM & $3,22 \times 10^{9}$ & 56,46 & 0,9977 \\
\hline & $\mathrm{Rp}$ & $1,02 \times 10^{10}$ & 59,42 & 0,9969 \\
\hline & EIS & $7,86 \times 10^{9}$ & 58,76 & 0,9905 \\
\hline & Promedio & $7,09 \times 10^{9}$ & 58,21 & \\
\hline \multirow[t]{4}{*}{0,50} & EFM & $2,27 \times 10^{8}$ & 50,34 & 0,9893 \\
\hline & $\mathrm{Rp}$ & $7,47 \times 10^{8}$ & 53,61 & 0.9997 \\
\hline & EIS & $8,59 \times 10^{8}$ & 54,05 & 0.9947 \\
\hline & Promedio & $6,11 \times 10^{8}$ & 52,67 & \\
\hline \multirow[t]{4}{*}{1,00} & EFM & $1,77 \times 10^{7}$ & 44,00 & 0.9930 \\
\hline & $\mathrm{Rp}$ & $1,32 \times 10^{8}$ & 49,50 & 0.9956 \\
\hline & EIS & $3,19 \times 10^{7}$ & 45,83 & 0.9984 \\
\hline & Promedio & $6,05 \times 10^{7}$ & 46,44 & \\
\hline
\end{tabular}

El valor promedio de $65,54 \mathrm{~kJ} / \mathrm{mol}$ obtenido para la $\mathrm{E}_{\mathrm{a}}{ }_{\mathrm{a}}$ del proceso de corrosión del acero A192 en $\mathrm{HCl} 0,5 \mathrm{M}$ (sin inhibidor) está en concordancia con lo reportado por POPOVA et al. [23], (61,33 kJ/mol) y ABD EL REHIM et al. [24], (61,50 kJ/mol). El aumento de los valores de eficiencia de inhibición con el incremento de la temperatura y los menores valores de $\mathrm{E}_{\text {a }}^{*}$ y A (factor pre-exponencial de la ecuación de Arrhenius) en presencia del ML, puede interpretarse que el ML fue quimisorbido en la superficie del acero A192. IVANOV, [25] considera el incremento de la eficiencia de inhibición con el aumento de la temperatura, como el cambio en la naturaleza del modo de adsorción; el inhibidor se adsorbe físicamente a temperaturas más bajas, mientras que la quimisorción se ve favorecida a medida que aumenta la temperatura. POPOVA et al. $[23,26]$ atribuyen la disminución de la $\mathrm{E}_{\mathrm{a}}^{*} \mathrm{y}$ A en presencia del inhibidor a la quimisorción del inhibidor en la superficie del acero.

\subsection{Morfología de la superficie}

La morfología superficial del acero A192 expuesto a soluciones de $\mathrm{HCl}$ 0,5 M en ausencia y presencia de 1,0 g/l ML después de 4 h y 24 h de inmersión a 298 K, se examinó usando microscopia electrónica de barrido. El análisis de las micrografías de la figura 9 revelan que la muestra de acero presenta una superficie muy rugosa en ausencia del ML (Fig. 9 a y b) como se esperaba debido al ataque corrosivo por la solución ácida. De acuerdo a la morfología del ataque y la microestructura del acero, el ataque corrosivo fue mayor en la fase ferrítica que en la perlita. En presencia del ML (figura 9 c y d) la superficie es comparativamente más lisa lo que indica el efecto inhibidor del ML. 

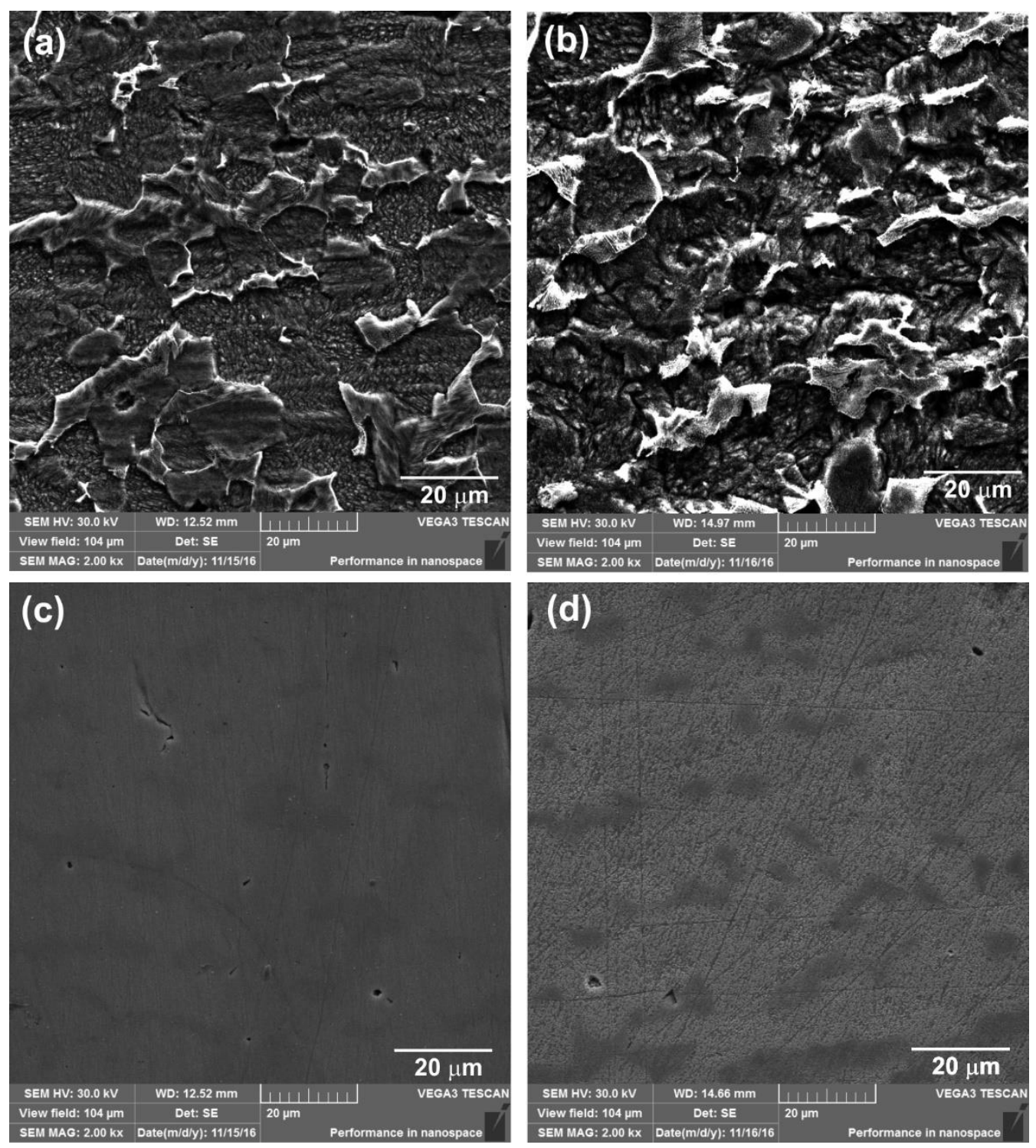

Figura 9: Micrografías electrónicas de barrido para el acero ASTM A192 expuesto en la solución HCl 0,5 M por (a) 4 h y (b) $24 \mathrm{~h}$, y expuesto en $\mathrm{HCl} 0,5 \mathrm{M}+1,0 \mathrm{~g} / \mathrm{l}$ de ML por (c) $4 \mathrm{~h}$ y (d) $24 \mathrm{~h}$, a $25^{\circ} \mathrm{C}$.

\section{CONCLUSIONES}

Se investigó el efecto del ML como inhibidor de la corrosión del acero ASTM A192 en HCl 0,5 M a diferentes temperaturas. Las siguientes conclusiones fueron obtenidas:

- El ML actúa como un óptimo inhibidor de la corrosión del acero ASTM A192 en HCl 0,5 M. La eficiencia de inhibición aumenta con la temperatura, obteniéndose eficiencias promedios con las técnicas EFM, Rp y EIS de $84,3 \%$ a $25^{\circ} \mathrm{C}, 89,0 \%$ a $45^{\circ} \mathrm{C}$ y $91,8 \%$ a $65^{\circ} \mathrm{C}$, para una concentración del ML de $1 \mathrm{~g} / \mathrm{l}$.

- El comportamiento de la adsorción del ML en la superficie del acero se ajustan a la isoterma de adsorción de Langmuir.

- Se encontró que la eficiencia de inhibición y la constante de equilibrio de adsorción incrementaron con el aumento de temperatura. La entalpia estándar de adsorción fue positiva y la energía de activación disminuyó con el incremento de la concentración del ML. Todos estos parámetros indican que la adsorción del ML en la superficie del acero A192 ocurre mediante una adsorción química.

- Los datos de polarización potenciodinámica indican que conforme incrementa la temperatura el comportamiento del ML pasa desde un inhibidor de tipo anódico a uno de tipo mixto.

- Las técnicas EFM, Rp y EIS utilizadas para evaluar el efecto inhibidor del ML mostraron una buena concordancia en los resultados. 


\section{AGRADECIMIENTOS}

Este proyecto fue financiado por el Consejo Nacional de Ciencia, Tecnología e Innovación Tecnológica CONCYTEC según convenio 120-2015-FONDECYT-DE. Los autores agradecen al Dr. Gustavo Sergio Duffó por la revisión del manuscrito.

\section{BIBLIOGRAFÍA}

[1] RAJA, P.B., SETHURAMAN, M.G., "Natural products as corrosion inhibitor for metals in corrosive media - A review", Materials Letters, v. 62, pp. 113-116, 2008.

[2] SANGEETHA, M., RAJENDRAN, S., MUTHUMEGALA, T.S., et al., "Green corrosion inhibitors-An Overview", Zaštita materijala, v. 52, pp. 3-19, 2011.

[3] PATNI, N., AGARWAL, S., SHAH, P., "Greener Approach towards Corrosion Inhibition", Chinese Journal of Engineering, v. 2013, Article ID 784186, 10 pages, 2013.

[4] FINSGAR, M., JACKSON, J., "Application of corrosion inhibitors for steels in acidic media for the oil and gas industry: A review", Corrosion Science, v. 86, pp. 17-41, 2014.

[5] SHIM, Y.Y., GUI, B., ARNISON, P.G., et al., "Flaxseed (Linum usitatissimum L.) bioactive compounds and peptide nomenclature: A review", Trends in Food Science \& Technology, v. 38, pp. 5-20, 2014.

[6] CUI, W., MAZZA, G., OOMAH, B.D., et al., "Optimization of an aqueous extraction process for flaxseed gum by response surface methodology", LWT - Food Science and Technology, v. 27, n. 4, pp. 363-369, 1994.

[7] CUI, W., MAZZA, G., BILIADERIS, C.G., "Chemical structure, molecular size distributions, and rheological properties of flaxseed gum”, Journal of Agricultural and Food Chemistry, v. 42, n. 9, pp. 1891-1895, 1994.

[8] BOSCH, R.W., HUBRECHT, J., BOGAERTS, W.F., et al., "Electrochemical frequency modulation: a new electrochemical technique for online corrosion monitoring", Corrosion, v. 57, n. 1, pp. 60-70, 2001.

[9] WU, X., MA, H., CHEN, S., et al., "General equivalent circuits for faradic electrode processes under electrochemical reaction control", Journal of the Electrochemical Society, v. 146, n. 5, pp. 1847-1853, 1999.

[10] KACURÁKOVÁ, M., CAPEK, P., SASINKOVÁ, V., et al., "FT-IR study of plant cell wall model compounds: pectic polysaccharides and hemicelluloses", Carbohydrate Polymers, v. 43, pp. 195-203, 2000.

[11] BARTH, A., "Infrared spectroscopy of proteins", Biochimica et Biophysica Acta, v. 1767, pp. 10731101, Jun. 2007.

[12] CUI, S.W., PHILLIPS, G.O., BLACKWELL, B., et al., "Characterisation and properties of Acacia senegal (L.) Willd. var. senegal with enhanced properties (Acacia (sen) SUPERGUM ${ }^{\mathrm{TM}}$ ): Part 4. Spectroscopic characterisation of Acacia senegal var. senegal and Acacia (sen) SUPERGUM ${ }^{\mathrm{TM}}$ Arabic, Food Hydrocolloids, v. 21, pp. 347-352, 2007.

[13] YAN, Y., LI, W., CAI, L., et al., "Electrochemical and quantum chemical study of purines as corrosion inhibitors for mild steel in $1 \mathrm{M} \mathrm{HCl}$ solution", Electrochimica Acta, v. 53, pp. 5953-5960, Apr. 2008.

[14] McCAFFERTY, E., "Validation of corrosion rates measured by the Tafel extrapolation method", Corrosion Science, v. 47, pp. 3202-3215, Sept. 2005.

[15] BOCKRIS, J.O., SWINKELS, D.A.J., “Adsorption of n-decylamine on solid metal electrodes", Journal of The Electrochemical Society, v. 111, n. 6, pp. 736-743, Jun. 1964.

[16] VRACAR, L.M., DRAZIC, D.M., "Adsorption and corrosion inhibitive properties of some organic molecules on iron electrode in sulfuric acid", Corrosion Science, v. 44, pp. 1669-1680, 2002.

[17] ZHAO, T., MU, G., "The adsorption and corrosion inhibition of anion surfactants on aluminium surface in hydrochloric acid", Corrosion Science, v. 41, pp. 1937-1944, 1999.

[18] BENTISS, F., LEBRINI, M., LAGRENÉE, M., "Thermodynamic characterization of metal dissolution and inhibitor adsorption processes in mild steel/2,5-bis(n-thienyl)- 1,3,4-thiadiazoles/hydrochloric acid system", Corrosion Science, v. 47, pp. 2915-2931, Aug. 2005.

[19] METIKOS-HUKOVIC, M., BABIC, R., et al., "Impedance investigation of corrosion inhibition of armco iron by thiourea", Journal of Applied Electrochemistry, v. 26, pp. 443-449, 1996.

[20] WANG, H-L., FAN, H-B., ZHENG, J-S., "Corrosion inhibition of mild steel in hydrochloric acid solution by a mercapto-triazole compound, Materials Chemistry and Physics, v. 77, pp. 655-661, 2002. 
[21] LI, X., TANG, L., LI, L., et al., "Synergistic inhibition between o-phenanthroline and chloride ion for steel corrosion in sulphuric acid", Corrosion Science, v. 48, pp. 308-321, 2006.

[22] DURNIE, W., MARCO, R., JEFFERSON, A., et al., "Development of a structure - activity relationship for oil field corrosion inhibitors", Journal of The Electrochemical Society, v.146, n. 5, pp. 1751-1756, 1999.

[23] POPOVA, A., SOKOLOVA, E., RAICHEVA, S., et al., "AC and DC study of the temperature effect on mild steel corrosion in acid media in the presence of benzimidazole derivatives", Corrosion Science, v. 45, pp. 33-58, 2003.

[24] ABD EL REHIM, S.S., IBRAHIM, M.A.M., et al., "The inhibition of 4-(2'-amino-5'-methylphenylazo) antipyrine on corrosion of mild steel in $\mathrm{HCl}$ solution", Materials Chemistry and Physics, v. 70, pp. 268-273, 2001.

[25] IVANOV, E.S., Inhibitors for Metal Corrosion in Acid Media, 1 ed., Metallurgy, 1986.

[26] POPOVA, A., "Temperature effect on mild steel corrosion in acid media in presence of azoles", Corrosion Science, v. 49, pp. 2144-2158, 2007. 